\title{
Vibration and skin blood flow changes in subjects with restless legs syndrome
}

\author{
This article was published in the following Dove Press journal: \\ Journal of Parkinsonism and Restless Legs Syndrome \\ 20 February 2014 \\ Number of times this article has been viewed
}

\section{Ulrike H Mitchell \\ Paula K Johnson}

Department of Exercise Sciences, Brigham Young University, Provo, UT, USA
Correspondence: Ulrike H Mitchell Brigham Young University, Department of Exercise Sciences, SFH \# 268,

Provo, UT 84602, USA

$\mathrm{Tel}+\mathrm{I} 80 \mathrm{I} 4223344$

Fax +l 80I 4220555

Email rike_mitchell@byu.edu
Objective: Vascular disturbances leading to tissue hypoxia have been named as one of the possible causes of restless legs syndrome (RLS). Whole body vibration (WBV) in healthy individuals results in nitric oxide (NO) generation, which then leads to increased blood flow. The purpose of this investigation was to determine if WBV can: 1) improve skin blood flow, as measured in flux, in individuals with RLS, and 2) induce increases in NO blood concentration. The data were compared to healthy age-matched subjects.

Design: Repeated measures, using two groups (RLS and control) that underwent two treatments (WBV) and sham (no vibration).

Setting: Data collection occurred in an institutional setting.

Patients: Ten subjects with RLS and ten controls.

Methods: Subjects underwent a ten bout, 30-second per bout WBV and one sham treatment session. Blood drawn for $\mathrm{NO}$ analysis and flux measurements with laser Doppler were performed before, immediately after, and 5 minutes after the sessions.

Main outcome measurements: The dependent variables, measured at three time intervals, were skin blood flow (flux) as determined by laser Doppler imaging and blood nitric oxide concentration.

Results: Baseline flux was significantly higher in the RLS group compared to control $(P<0.001)$; flux in the RLS group immediately after WBV was significantly higher compared to baseline $(P<0.05)$, sham treatment $(P<0.05)$, and control group $(P<0.05)$. There was no difference in NO concentration within subjects and between groups.

Conclusion: Subjects with RLS have higher skin blood flow than controls and a greater increase in flux with WBV without concurrent increases in NO concentration from blood drawn at the antecubital fossa.

Keywords: restless legs syndrome, flux, SBF, WBV, vascular disturbance

\section{Introduction}

Restless legs syndrome (RLS) is a chronic sensorimotor disorder, ${ }^{1}$ characterized by a strong urge to move, accompanied or caused by uncomfortable, or even distressing paresthesia of the legs, described as a "creeping, tugging, pulling" feeling. ${ }^{2}$ The symptoms often become worse as the day progresses, leading to sleep disturbances or sleep deprivation and hence to strong fatigue, tiredness, and low energy during the day time. ${ }^{2}$ The symptoms are usually lessened by movement. ${ }^{2}$

There are several hypotheses concerning the etiology of RLS. One of the first modern-day (1940s and 1950s) theories is that the symptoms are the result of decreased 


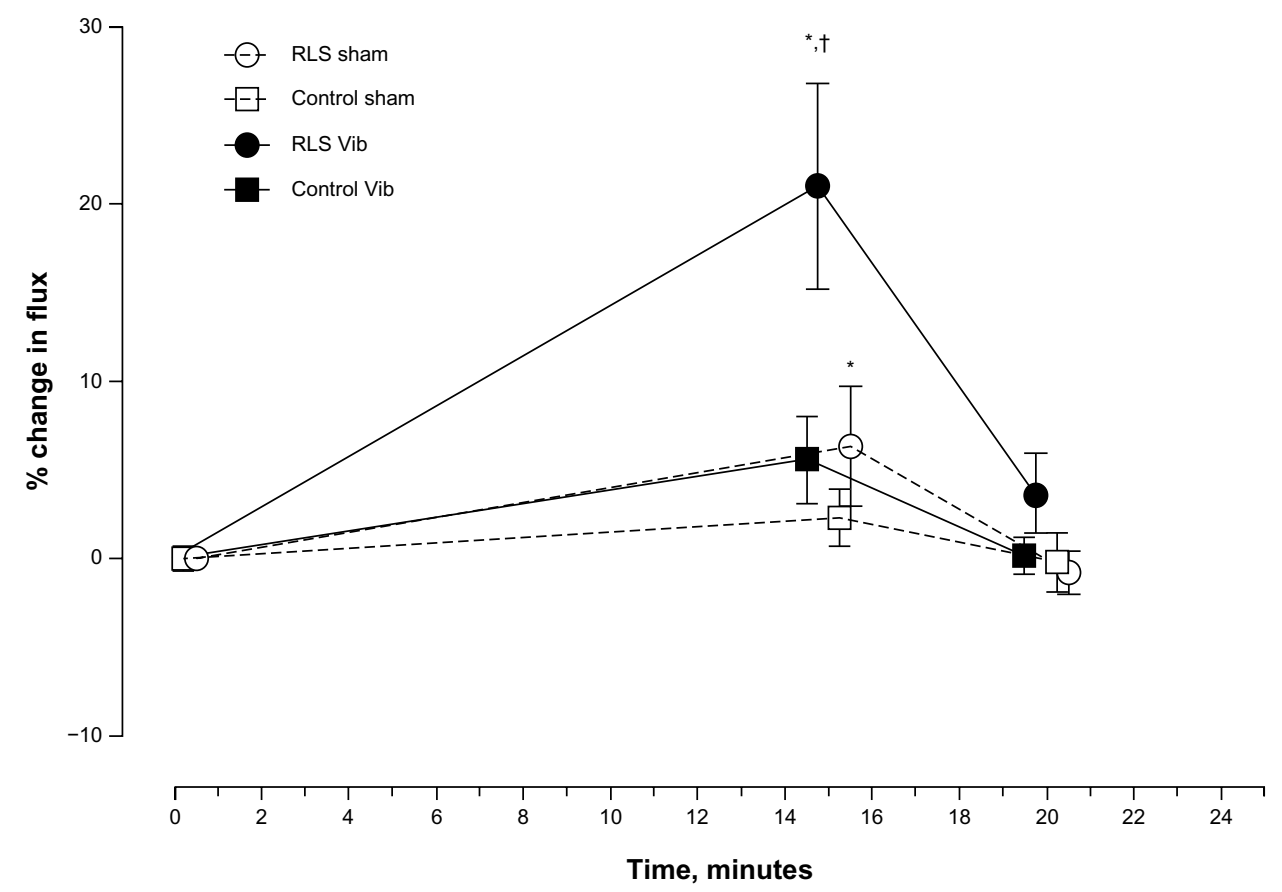

Figure I Average percentage change in flux with standard error of the mean bars.

Notes: *Significant difference between RLS treatment and sham; ${ }^{\dagger}$ significant difference to all other points.

Abbreviations: RLS, restless legs syndrome; Vib, vibration.

tissue perfusion. ${ }^{3}$ The urge to move may be a subconsciously driven mechanism to augment blood flow and tissue perfusion. In 1985 this theory came under disfavor when it was observed that patients with RLS responded well to dopaminergic agents, such as Levodopa, and dopamine agonists. ${ }^{4,5}$
Due to its unclear pathogenesis, the treatment of RLS has been the subject of debate for many years, and has fragmented researchers and sufferers alike into three groups: one trying to manage the symptoms by changing lifestyle; one addressing dopamine regulation in the central

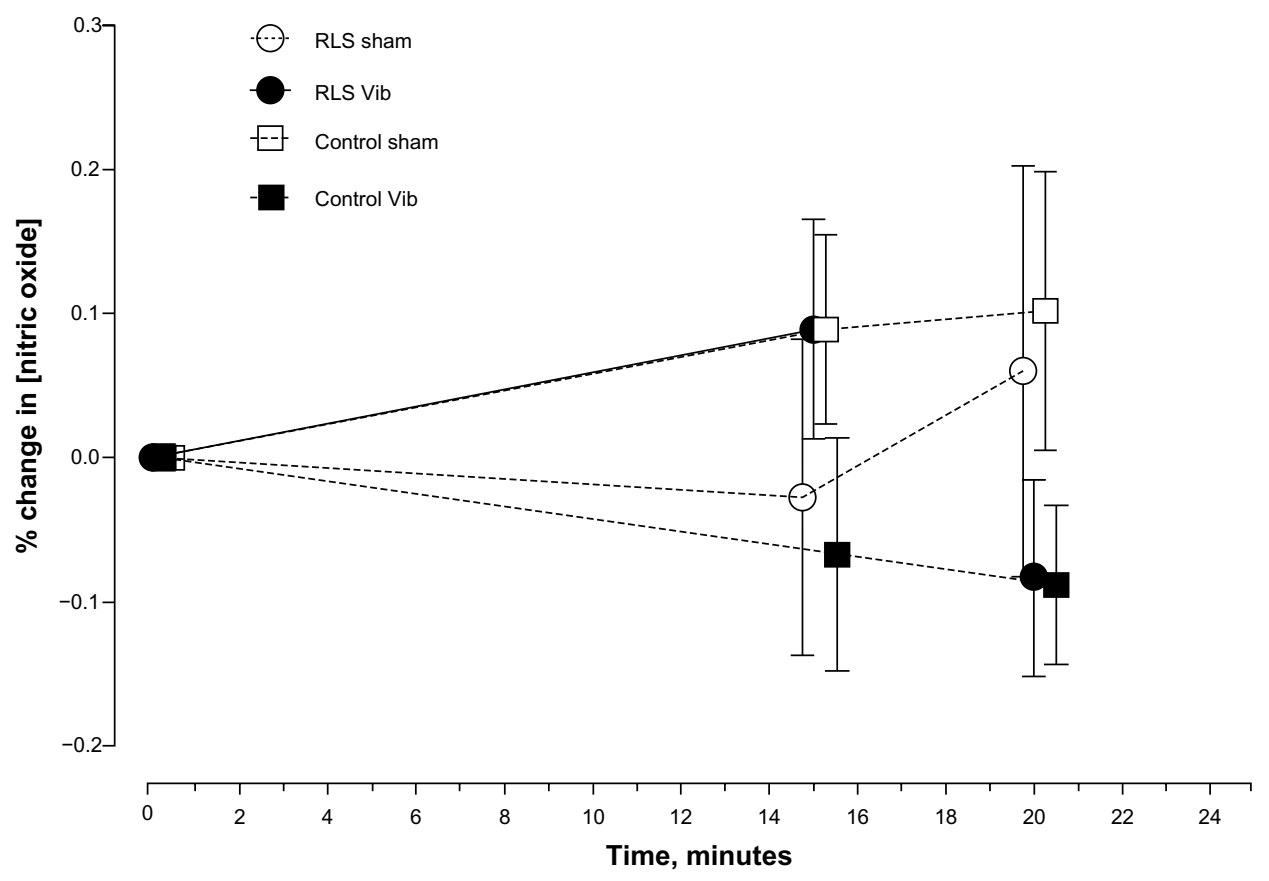

Figure 2 Average percentage change in nitric oxide with standard error of the mean bars. Abbreviations: RLS, restless legs syndrome; Vib, vibration. 
nervous system via pharmaceuticals; and one tackling the peripheral nervous system and blood flow. The latter is of special interest to those who have experienced side effects to drugs. The theory behind that line of research is based on the historical model that decreased blood flow leads to hypoxia, which in turn is at least partially responsible for the characteristic unpleasant sensations and the urge to move. ${ }^{3}$ Almost a half a century after Ekbom's findings, ${ }^{2}$ other research has shown that enhanced circulation decreases RLS symptoms. Modalities, such as pulsed compression devices ${ }^{3,6,7}$ massage, ${ }^{8}$ and near-infrared light (NIR) ${ }^{9-11}$ which improve blood flow, have been used in the treatment of RLS. Admittedly, the above modalities also provide sensory stimulation, which could equally be responsible for any benefits seen with regard to RLS symptoms. However, the vascular theory is strengthened by the fact that physical activity involving movement of the legs is one of the few non-pharmacological treatment options available to RLS sufferers, and epidemiologic evidence exists indicating that lack of exercise is a strong predictor of and a significant risk factor for RLS. ${ }^{12}$ In fact, by definition, RLS is the urge to move that is at least partially relieved by movement. ${ }^{13}$ Limb movement increases blood flow, ${ }^{14}$ which increases the shear stress between the inner wall of the endothelium and the moving blood. This stress, or shear force, stimulates the generation of nitric oxide (NO). ${ }^{15,16} \mathrm{NO}$ then initiates a cascade of events that ultimately leads to the relaxation of smooth muscles in the blood vessel and consequently to further increased local blood flow. ${ }^{17-19}$

Investigations have shown that externally applied low frequency oscillation or vibration to the whole body in healthy individuals results in endothelial shear stress ${ }^{20}$ sufficient to produce $\mathrm{NO},{ }^{21}$ which then leads to increased skin blood flow ${ }^{22}$ and augmented muscle blood volume. ${ }^{23}$ Research has yet to be conducted to determine if individuals with RLS respond to whole body vibration (WBV) with enhanced circulation as well. The results could potentially be another piece in the pathogenesis puzzle of RLS.

The purpose of this investigation was twofold: to determine if WBV can improve skin blood flow in individuals with RLS and induce increases in blood concentration of nitric oxide in these individuals. The data were compared to healthy age-matched subjects.

\section{Methods and procedures}

This study was approved by the university's institutional review board. According to a power analysis, which was performed a priori using data obtained from studies conducted with healthy adults, it was determined that we needed nine subjects to show a difference in blood flow at a significance level of 0.05 . Ten subjects (Table 1) with RLS (male [M]:female [F] 5:5) and ten age-matched subjects without RLS (M:F 5:5) were recruited for this study via newspaper advertisement, flyer, and word of mouth. Other research with a similar set up has used the same number of subjects. ${ }^{24}$

In an attempt to exclude patients with secondary RLS we screened the patients during an interview by asking them if they were pregnant, had iron deficiency anemia, or if they had been diagnosed with diabetes. Patients who tested positive for peripheral neuropathy and deep vein thrombosis were excluded from this study. Candidates were also excluded if they have had any injury or surgery to their lower extremities within the past three months, had any known cardiovascular disease, or were taking any nitric oxide donors.

All subjects visited our laboratory twice: once for the vibration condition and the other visit for the control/sham condition. Between each visit there was a washout period of no less than 2 days. The order of these conditions was blocked to avoid a treatment order effect. The first visit required 60 minutes and included participant screening procedures and signing the informed consent form, while the second visit required 30 minutes. The data collection for all subjects happened between the hours of $1 \mathrm{pm}$ and $4 \mathrm{pm}$.

On the first visit, subjects reported to the lab to complete pre-participation questionnaires, including confirmation of the RLS diagnosis by asking the five essential diagnostic criteria. ${ }^{25}$ RLS severity was measured by the International Restless Legs Syndrome Rating Scale (IRLS). Quantitative sensory testing (QST) for heat and cold sensation thresholds was performed in order to ascertain and consequently to exclude subjects with signs of neuropathy in small A-delta and C-nerve fibers. Thermal sensory thresholds were quantified using a TSAII

Table I Subject characteristics

\begin{tabular}{|c|c|c|c|c|c|c|}
\hline Group & $\begin{array}{l}\text { Age } \\
\text { (years) }\end{array}$ & $\begin{array}{l}\text { Weight } \\
\text { (kg) }\end{array}$ & $\begin{array}{l}\text { Height } \\
(\mathrm{cm})\end{array}$ & $\begin{array}{l}\text { Body mass } \\
\text { index }\left(\mathrm{kg} / \mathrm{m}^{2}\right)\end{array}$ & $\begin{array}{l}\text { Years since RLS } \\
\text { diagnosis }\end{array}$ & $\begin{array}{l}\text { IRLS } \\
\text { score }\end{array}$ \\
\hline RLS & $44.6 \pm 13.6$ & $78.4 \pm 13.2$ & $171.6 \pm 11.6$ & 26.8 & $11.3 \pm 4.3$ & $19.7 \pm 7.5$ \\
\hline Control & $43.8 \pm 15.3$ & $74.1 \pm 10.8$ & $170.1 \pm 9.1$ & 25.5 & $\mathrm{n} / \mathrm{a}$ & $\mathrm{n} / \mathrm{a}$ \\
\hline
\end{tabular}

Abbreviations: IRLS, International Restless Legs Syndrome Rating Scale; RLS, restless legs syndrome. 
Neurosensory Analyzer (Medoc Ltd, Ramat Yishai, Israel) using the method of limits procedure. ${ }^{26}$

A compression ultrasound (portable ultrasound system; GE Healthcare, Little Chalfont, UK) was performed bilaterally on the common femoral and the popliteal veins to screen for possible deep vein thrombosis. This was done in order to rule out the threat of dislodging a clot during vibration.

Upon meeting all inclusion criteria and signing the informed consent form, the subjects were escorted to the environmental chamber that is controlled at $21^{\circ} \mathrm{C}-22^{\circ} \mathrm{C}$. They were seated in a semi-recumbent position in a dental chair with their bare feet resting on a platform to ensure a $90^{\circ}$ angle at the knees. After a 5-minute acclimatization period, a licensed phlebotomist inserted a venous catheter in a large antecubital vein and a $3 \mathrm{~mL}$ sample of blood was drawn and collected into a standard ethylenediaminetetraacetic acid (EDTA) vacutainer tube. A preservative solution consisting of $0.8 \mathrm{M}$ ferricyanide, $0.1 \mathrm{M} \mathrm{N}$-ethylmaleimide, and Nonidet P-40 was added to the blood in a 4:1 (volume:volume; whole blood:preservative) ratio. The sample was then stored at $-80^{\circ} \mathrm{C}$ for future analysis.

Following the first blood draw, a laser Doppler image (LDI; Moor Instruments Inc., Oxford, UK) of the dorsum of the right foot was captured. The scan area was marked to ensure consistency between scans, which was set to $5 \mathrm{~cm}$ by $5 \mathrm{~cm}$. The resolution was $100 \times 100$ pixels, and the scan rate was $4 \mathrm{~ms} /$ pixel. The resulting images were evaluated in processing software (Moor LDI Processing V3.1, Moor Instruments Inc). After donning their shoes, the subjects transferred onto the vibration platform, also located in the chamber. The subjects then underwent the vibration treatment or sham treatment as dictated by coin toss.

\section{Vibration treatment}

The subject stood on the vibration platform with knees flexed to about $30^{\circ}$, their weight centered over the middle of each foot. This stance was used in order to attenuate the vibrations to the spine. The feet were approximately $25 \mathrm{~cm}$ apart. Ten 30-second bouts of WBV at a frequency and amplitude of $26 \mathrm{~Hz}$ and $2 \mathrm{~mm}$, respectively, were performed, with a 1 -minute rest while standing between each bout. Following the last bout, the subject returned to the seat.

\section{Sham treatment}

The subject stood on the vibration platform assuming the same position as for the vibration treatment, for the same intervals, but no vibration stimulus was given.
Following the treatment and 5 minutes post treatment, second and third $3 \mathrm{~mL}$ blood draws were performed; immediately following each blood draw LDI scans were done. The blood samples were preserved and frozen. The catheter was removed and its insertion site was cleaned and covered with gauze to ensure compliance with infection control procedures.

On the second visit, the subjects reported directly to the environmental chamber, where they were seated with their right shoe and sock off to become acclimatized. The same procedure as described previously was followed, using the appropriate treatment (vibration or sham).

\section{Skin blood flow measurements}

The laser Doppler imager determines the velocity of red blood cells in arterioles, venules, and capillaries 1-4 mm under the skin surface. The output is the "flux" of red blood cells, defined as the number of red blood cells times their velocity, which determines circulation. ${ }^{27}$ The imager enables rapid non-invasive analysis of changes in blood flow in skin and correlates well with other methods for measuring skin perfusion. ${ }^{28,29}$

\section{Blood analysis}

For blood NO concentrations analysis, we used the Sievers Nitric Oxide Analyzer (model 280i, GE Analytical Instruments, Boulder, CO, USA). The blood was thawed, deproteinated, and centrifuged. Triplicates of $10 \mu \mathrm{L}$ aliquots of each blood sample were injected into the $\mathrm{NO}$ analyzer purge vessel following the protocol by Pelletier et al. ${ }^{30}$ Data acquisition software connected to the NO analyzer was used to convert detected light resulting from the chemiluminescent reaction into a voltage signal and record the subsequent tracings. Data analysis software integrated the tracings peak by peak to obtain the area under the curve and report it as an NO concentration.

\section{Statistical analysis}

This repeated measures study design used two groups (RLS and control) that underwent two treatments (WBV) and sham (no vibration). The dependent variables were skin blood flow (flux) as measured by laser Doppler imaging on the dorsum of the right foot before treatment ("0 minutes"), immediately after treatment ("15 minutes"), and 5 minutes after treatment ("20 minutes"), and blood NO concentration, measured at the same time intervals.

\section{Flux}

A $2 \times 2 \times 3($ group $\times$ treatment $\times$ time $)$ mixed model analysis of variance (ANOVA) was performed, followed by the Tukey's 
multiple comparisons test (GraphPad Prism version 6.0 for Mac, GraphPad Software, La Jolla, CA, USA). The dependent variable was the mean flux, the independent variables were the groups (individuals with RLS and controls), treatments (vibration and sham), and time (pre, post and 5 minutes post). Alpha was set at 0.05 .

\section{NO production}

A $2 \times 2 \times 3($ group $\times$ treatment $\times$ time $)$ mixed model ANOVA was performed. The dependent variable was NO content in venous blood, the independent variables were the groups (individuals with RLS and controls), treatments (vibration and sham), and time (pre, post and 5 minutes post).

\section{Results}

None of the subjects were smokers. Six of the subjects had a family history of RLS; two of the subjects without family history of RLS had been diagnosed with RLS when in their teens and twenties, allowing us to infer that they are likely to have primary RLS; ${ }^{31}$ the remaining two subjects started having symptoms in their late 40's and early 50's, but did not display any obvious causes for secondary RLS, as ascertained during the initial screen. Two subjects were taking ropinirole and one subject was taking lorazepam for his RLS-related sleep deprivation.

\section{Flux}

There was a significant difference in baseline flux between the RLS and control groups ( $P<0.001)$, with RLS having the greater flux. In order to account for the differences in baseline flux we used percent change for subsequent analyses:

$$
\text { [(flux - baseline flux)/baseline flux] }
$$

There was an interaction between the variables $(P=0.0004)$; there was a significant difference in flux between the three time points (baseline, immediately after treatment [about 15 minutes] and 5 minutes post-treatment [about 20 minutes; $P<0.0001]$ ), between the treatments (vibration and sham; $P=0.0056$ ) and between groups (RLS and control; $P=0.043$ ) (Figure 1). Post hoc analyses using Tukey's honest significant difference (HSD), with an alpha set at 0.05 , indicated that the flux in the RLS group immediately after vibration was significantly different to any other flux within subjects and between groups.

\section{NO content}

There was no difference in baseline concentration (RLS average $=45.7$, standard error of the mean $[$ SEM] 9.6, stan- dard deviation [SD] 27.0; control average =51.9, SEM 3.1, SD $6.9 ; P=0.8)$. In order to account for the great variability, especially in the RLS group and in order to compare this analysis to the flux, we used percent change for consequent analyses:

[(NO concentration - baseline NO concentration)/ baseline NO concentration]

There was no interaction $(P=0.53)$, no difference in venous blood NO content between the different time points ( $P=0.89$ ), groups $(P=0.14)$, and treatment $(P=0.61)$ (Figure 2$)$.

\section{Discussion}

The purpose of this investigation was to determine if a ten $\times$ 30 -second bout of WBV improved skin blood flow and induced increases in NO blood concentration in individuals with RLS compared to healthy age-matched subjects, in order to potentially find a non-invasive, non-pharmaceutical means to treat the symptoms associated with RLS. This notion was anchored by the findings of earlier research that showed that increased blood flow diminished RLS symptoms. ${ }^{2,3,6-11}$

\section{Flux}

There was a significant increase in skin blood flow immediately after the ten $\times 30$-second bout of WBV in the RLS group compared to baseline, its 5-minute post treatment flux, its sham treatment data, as well as when compared to any time point in the control group. No other comparisons were found to be at a level of significance. These findings were surprising for several reasons: the flux in the RLS group immediately after vibration increased significantly compared to baseline. We had anticipated a blunted flux response based on our (and historical) hypothesis that RLS sufferers have the urge to move in order to increase blood flow - possibly due to endothelial dysfunction. This study did not confirm our hypothesis; in fact, at first sight one might surmise that it was repudiated. The significant increase in flux immediately after vibration demonstrates an excellent ability for RLS sufferers to augment skin blood flow, albeit possibly delayed. One needs to consider that all data were collected in the early afternoon and not at night, when the urge to move usually becomes prominent or worsens. It is conceivable that subjects with RLS exhibit sufficient blood flow and possess good vasodilating ability during the day until the evening, following a circadian rhythm, when tissue hypoxia settles in due to vasoconstriction, possibly via sympathetic nervous system. Other studies ${ }^{32,33}$ have implicated 
sympathetic nervous system hyperactivity in the involvement of symptoms associated with RLS.

Two of the subjects were taking dopamine agonists at the time of data collection. Dopamine has an effect on blood flow $^{34}$ and could potentially have influenced the flux we measured. However, we assessed the flux data from the two subjects who were taking dopamine agonists and found that, while one subject exhibited above-average flux at baseline, the other subject displayed below-average flux at baseline. This suggests that the intake of dopamine agonists might not have had an impact on the outcome.

The second reason why we found our results surprising was that the flux in our control group did not increase significantly when comparing pre- to post-treatment data. Other research has shown that flux increased by over $400 \%$ in the forearm after a 5-minute vibration treatment in healthy older adults. ${ }^{35}$ However, in that study, the vibration was applied merely locally as only the forearm was vibrated and the amplitude of oscillation was 5-6 mm, up to three times the vertical displacement compared to our study. The decrease in weight being vibrated and the fact that the distal end of the vibrating arm was not anchored, could very well have had an influence on the amount of vibrational impact and thus shear force generation on the blood vessels. There exists a direct relationship between the amount of shear force and elicited flow-mediated dilation. ${ }^{36} \mathrm{In}$ an attempt to accommodate for the very short half life of $\mathrm{NO},{ }^{37}$ we set up our protocol such that we drew blood for NO analysis immediately after the treatment and then started the Doppler scan. The fact that we didn't measure blood flow immediately after the treatment, but waited for a short period of time, could have been responsible for our lack of significant findings. Other research ${ }^{24}$ has found that flux in the common femoral artery returned to baseline 75 seconds after vibration bouts. This makes the significant increase in flux we saw in the patients with RLS the more remarkable, and it begs the following questions: is the flux response to WBV in patients with RLS delayed, is it stronger, or both?

The RLS group had a higher flux at baseline compared to the control group. This finding seems to confirm or can be explained by findings of another recent study, ${ }^{38}$ which assessed microvascular skin changes in patients with RLS. The results demonstrated an increased skin blood flow in the feet when compared to healthy controls and an impaired spatial cooling ability when environmental temperature was raised. The authors conclude that their data might support the notion that RLS patients suffer from some sort of sympathetic dysfunction since it controls skin microvascular circulation. Larsson et a ${ }^{39}$ made a similar discovery when they found higher tortuosity (length of capillary/perimeter of fiber) in the anterior tibialis in patients with RLS. The authors hypothesized that this alteration in microvessel arrangement could be the result of an attempt to counteract local hypoxia in the tissue. ${ }^{39}$

\section{NO content}

There was no difference in NO concentration between groups at baseline nor any other differences within or between groups. Therefore, our study did not confirm findings from the only other study ${ }^{40}$ where serum NO levels in RLS patients were measured. Baskol et $\mathrm{al}^{40}$ found serum NO levels to be lower in patients with RLS compared to healthy controls. While the average NO concentration was lower in our RLS group, its high variability, as expressed in the high standard deviation, makes a statistical comparison difficult. There are differences between that study and ours, but neither points to a definite cause for the difference in findings. Baskol et al's study used serum, while we used whole blood for the analysis; they used a one-step assay with nitrate reductase, while we used chemiluminescent reaction in order to determine NO content; our project, including blood draws, was performed in the afternoon, but it is not clear when their blood draw occurred. Furthermore, in our study the average IRLS score was 19.7, while Baskol et al do not indicate patient RLS severity.

There are several possible explanations for why there was an increase in flux in the foot without an accompanying increase in blood NO concentration following WBV. For instance, $\mathrm{NO}$ is not the only endogenous factor able to induce vasodilation and increases in blood flow. Other vasodilators, such as epinephrine, norepinephrine, or histamine, could have been activated through vibration and elicited a vasodilatory response. Di Loreto et $\mathrm{al}^{41}$ found increases in norepinephrine, but not epinephrine, after ten vibration series of 1-minute duration and 1-minute rest between each in ten healthy men. A similar increase was found in the control group, which, comparable to our study, also stood on the vibration platform, but was not vibrated. Since standing is one of the stimuli for norepinephrine release, the authors expected the increase in both groups. Norepinephrine plasma concentration returned to baseline in the control group, but not in the treatment group after resting for 30 minutes in a sitting position.

Another explanation for an increase in flux without an accompanying increase in blood $\mathrm{NO}$ concentration is that there was an increase of $\mathrm{NO}$ induced by shear stress through vibration, but only locally in the feet. In our study, blood 
samples for NO concentration measurements were drawn from the antecubital fossa, relatively far removed from where the skin blood flow measurements were made. Other studies, ${ }^{35,42}$ where blood samples for the NO assessment were drawn at the same location as where the vibration was applied, found NO increases alongside with increases in flux after vibration. This notion is strengthened by the fact that the subjects in our study were in a squatting position during vibration, which attenuated its effect in the upper extremity and hence, NO may not have been induced in the arm.

\section{Conclusion}

Skin blood flow, measured on the dorsum of the foot, was significantly higher in subjects with RLS compared to age-matched healthy subjects. Immediately following ten 30 -second bouts of WBV at a frequency of $26 \mathrm{~Hz}$ and amplitude of $2 \mathrm{~mm}$, skin blood flow increased significantly more in subjects with RLS compared to baseline and to control subjects. While this discrepancy in flux between subjects with and without RLS does not prove causation, it does clearly raise the possibility that there might be an association. NO concentration from blood drawn at the antecubital fossa did not change, perhaps due to attenuation of vibration at that location, or perhaps because increase in NO due to shear stress is only a local effect.

Further research should be conducted to clarify if subjects with RLS indeed react differently to vibration in the evening or early night. Blood flow in subjects with RLS should be measured during and immediately after WBV and compared to controls. Leg muscle blood flow should be measured to assess its change with treatment and influence on RLS symptoms. The amount of other vasodilators and vasoconstrictors should be measured and compared to healthy subjects. In addition, activity of the autonomic nervous system, especially the sympathetic component, should be assessed.

\section{Disclosure}

The authors disclose no conflicts of interest in this work.

\section{References}

1. Barrière G, Cazalets JR, Bioulac B, Tison F, Ghorayeb I. The restless legs syndrome. Prog Neurobiol. 2005;77(3):139-165.

2. Ekbom KA. Restless legs syndrome. Neurology. 1960;10:868-873.

3. Rajaram SS, Shanahan J, Ash C, Walters AS, Weisfogel G. Enhanced external counter pulsation (EECP) as a novel treatment for restless legs syndrome (RLS): a preliminary test of the vascular neurologic hypothesis for RLS. Sleep Med. 2005;6(2):101-106.

4. Ondo WG, Vuong KD, Jankovic J. Exploring the relationship between Parkinson disease and restless legs syndrome. Arch Neurol. 2002;59(3): 421-424.
5. Teive HA, Munhoz RP, Barbosa ER. Professor Karl-Axel Ekbom and restless legs syndrome. Parkinsonism Relat Disord. 2009;15(4): 254-257.

6. Lettieri CJ, Eliasson AH. Pneumatic compression devices are an effective therapy for restless legs syndrome: a prospective, randomized, double-blinded, sham-controlled trial. Chest. 2009;135(1):74-80.

7. Eliasson AH, Lettieri CJ. Sequential compression devices for treatment of restless legs syndrome. Medicine (Baltimore). 2007;86(6):317-323.

8. Russell M. Massage therapy and restless legs syndrome. KBodyw Mov Ther. 2007;11(2):146-150.

9. Mitchell UH, Myrer JW, Johnson AW, Hilton SC. Restless legs syndrome and near-infrared light: An alternative treatment option. Physiother Theory Pract. 2011;27(5):345-351.

10. Mitchell UH, Johnson AW, Myrer B. Comparison of two infrared devices in their effectiveness in reducing symptoms associated with RLS. Physiother Theory Pract. 2011;27(5):352-359.

11. Mitchell UH. Use of near-infrared light to reduce symptoms associated with restless legs syndrome in a woman: a case report. JMed Case Rep. 2010;4:286.

12. Phillips B, Young T, Finn L, Asher K, Hening WA, Purvis C. Epidemiology of restless legs symptoms in adults. Arch Intern Med. 2000;160(14):2137-2141.

13. Allen RP, Picchietti D, Hening WA, Trenkwalder C, Walters AS, Montplaisi J; Restless Legs Syndrome Diagnosis and Epidemiology workshop at the National Institutes of Health; International Restless Legs Syndrome Study Group. Restless legs syndrome: diagnostic criteria, special considerations, and epidemiology. A report from the restless legs syndrome diagnosis and epidemiology workshop at the National Institutes of Health. Sleep Med. 2003;4(2):101-119.

14. Clifford PS, Hellsten Y. Vasodilatory mechanisms in contracting skeletal muscle. J Appl Physiol (1985). 2004;97(1):393-403.

15. Burke TJ. 5 Questions - and answers - about MIRE treatment. $A d v$ Skin Wound Care. 2003;16(7):369-371.

16. Buga GM, Gold ME, Fukuto JM, Ignarro LJ. Shear stress-induced release of nitric oxide from endothelial cells grown on beads. Hypertension. 1991;17(2):187-193.

17. Ignarro LJ, Buga GM, Wood KS, Byrns RE, Chaudhuri G. Endotheliumderived relaxing factor produced and released from artery and vein is nitric oxide. Proc Natl Acad Sci U S A. 1987;84(24):9265-9269.

18. Moncada S, Palmer RM, Higgs EA. Nitric oxide: physiology, pathophysiology, and pharmacology. Pharmacol Rev. 1991;43(2): 109-142.

19. Ballermann BJ, Dardik A, Eng E, Liu A. Shear stress and the endothelium. Kidney Int Suppl. 1998;67:S100-S108.

20. Mester J, Kleinöder H, Yue Z. Vibration training: benefits and risks. J Biomech. 2006;39(6):1056-1065.

21. Sackner MA, Gummels E, Adams JA. Nitric oxide is released into circulation with whole-body, periodic acceleration. Chest. 2005;127(1): 30-39.

22. Lohman EB, Petrofsky JS, Maloney-Hinds C, Betts-Schwab H, Thorpe D. The effect of whole body vibration on lower extremity skin blood flow in normal subjects. Med Sci Monit. 2007;13(2):CR71-CR76.

23. Kerschan-Schindl K, Grampp S, Henk C, et al. Whole-body vibration exercise leads to alterations in muscle blood volume. Clin Physiol. 2001;21(3):377-382.

24. Lythgo N, Eser P, de Groot P, Galea M. Whole-body vibration dosage alters leg blood flow. Clin Physiol Funct Imaging. 2009;29(1):53-59.

25. International Restless Legs Syndrome Study Group. 2011 Revised IRLSSG Diagnostic Criteria for RLS [webpage on the Internet]. Rochester: International Restless Legs Syndrome Study Group; 2011. Available from: http://irlssg.org/diagnostic-criteria/. Accessed on January 7, 2014.

26. Chong PS, Cros DP. Technology literature review: quantitative sensory testing. Muscle Nerve. 2004;29(5):734-747.

27. Aubee AP. Laser Doppler Flowmetry. 2006. Available from: http:// www.ele.uri.edu/courses/ele482/S06/Andrew_1.pdf. Accessed January 7, 2014. 
28. Millet C, Roustit M, Blaise S, Cracowski JL. Comparison between laser speckle contrast imaging and laser Doppler imaging to assess skin blood flow in humans. Microvasc Res. 2011;82(2):147-151.

29. Essex TJ, Byrne PO. A laser Doppler scanner for imaging blood flow in skin. J Biomed Eng. 1991;13(3):189-194.

30. Pelletier MM, Kleinbongard P, Ringwood L, et al. The measurement of blood and plasma nitrite by chemiluminescence: pitfalls and solutions. Free Radic Biol Med. 2006;41(4):541-548.

31. Allen RP, Earley CJ. Defining the phenotype of the restless legs syndrome (RLS) using age-of-symptom-onset. Sleep Med. 2000;1(1):11-19.

32. Ware JC, Blumoff R, Pittard JT. Peripheral vasoconstriction in patients with sleep related periodic leg movements. Sleep. 1988;11(2): $182-186$.

33. Ancoli-Israel S, Seifert AR, Lemon M. Thermal biofeedback and periodic movements in sleep: patients' subjective reports and a case study. Biofeedback Self Regul. 1986;11(3):177-188.

34. Ishiguro A, Suzuki K, Sekine T, et al. Effect of dopamine on peripheral perfusion in very-low-birth-weight infants during the transitional period. Pediatr Res. 2012;72(1):86-89.

35. Maloney-Hinds C, Petrofsky JS, Zimmerman G, Hessinger DA. The role of nitric oxide in skin blood flow increases due to vibration in healthy adults and adults with type 2 diabetes. Diabetes Technol Ther. 2009;11(1):39-43.
36. Pyke KE, Tschakovsky ME. The relationship between shear stress and flow-mediated dilatation: implications for the assessment of endothelial function. J Physiol. 2005;568(Pt 2):357-369.

37. Liu X, Miller MJ, Joshi MS, Sadowska-Krowicka H, Clark DA, Lancaster JR. Diffusion-limited reaction of free nitric oxide with erythrocytes. J Biol Chem. 1998;273(30):18709-18713.

38. Anderson KN, Di Maria C, Allen J. Novel assessment of microvascular changes in idiopathic restless legs syndrome (Willis-Ekbom disease). J Sleep Res. 2013;22(3):315-321.

39. Larsson BW, Kadi F, Ulfberg J, Aulin KP. Skeletal muscle morphology in patients with restless legs syndrome. Eur Neurol. 2007;58(3): 133-137.

40. Baskol G, Korkmaz S, Erdem F, Caniklioglu A, Kocyigit M, Aksu M. Assessment of nitric oxide, advanced oxidation protein products, malondialdehyde, and thiol levels in patients with restless legs syndrome. Sleep Med. 2012;13(4):414-418.

41. Di Loreto C, Ranchelli A, Lucidi P, et al. Effects of whole-body vibration exercise on the endocrine system of healthy men. $J$ Endocrinol Invest. 2004;27(4):323-327.

42. Ichioka S, Yokogawa H, Nakagami G, Sekiya N, Sanada H. In vivo analysis of skin microcirculation and the role of nitric oxide during vibration. Ostomy Wound Manage. 2011;57(9):40-47.
Journal of Parkinsonism \& Restless Legs Syndrome

\section{Publish your work in this journal}

Journal of Parkinsonism and Restless Legs Syndrome is an online, open access, peer-reviewed journal. The journal publishes review articles, historical reviews, original research articles, case reports, letters to the editor, clinical teaching cases, neuroradiology highlights, neuropathology highlights, neuropsychiatry highlights, autobiographies, conference

\section{Dovepress}

proceedings, abstracts and book reviews. The manuscript management system is completely online and includes a very quick and fair peerreview system, which is all easy to use. Visit http://www.dovepress.com testimonials.php to read real quotes from published authors. 